Published in final edited form as:

Cancer Res. 2021 March 15; 81(6): 1472-1485. doi:10.1158/0008-5472.CAN-20-2383.

\title{
Serine-threonine kinase TAO3-mediated trafficking of endosomes containing the invadopodia scaffold TKS5a promotes cancer invasion and tumor growth
}

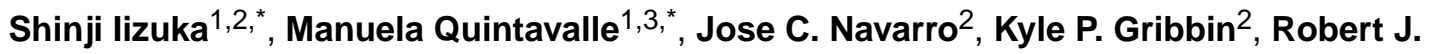 \\ Ardecky $^{1}$, Matthew M. Abelman ${ }^{1}$, Chen-Ting Ma ${ }^{1}$, Eduard Sergienko ${ }^{1}$, Fu-Yue Zeng ${ }^{1}$, lan \\ Pass $^{1}$, George V. Thomas ${ }^{4}$, Shannon K. McWeeney ${ }^{4,5,6}$, Christian A. Hassig ${ }^{1,7}$, Anthony B. \\ Pinkerton $^{1,7}$, Sara A. Courtneidge e $^{1,2,4,8,9}$ \\ ${ }^{1}$ Sanford Burnham Prebys Medical Discovery Institute, La Jolla, CA, USA \\ ${ }^{2}$ Department of Cell Developmental \& Cancer Biology, Oregon Health \& Science University, \\ Portland, OR, USA \\ ${ }^{3}$ present address: AstraZeneca, Milan, Italy \\ ${ }^{4}$ Knight Cancer Institute, Oregon Health \& Science University, Portland, OR, USA \\ ${ }^{5}$ Division of Bioinformatics and Computational Biology, Department of Medical Informatics and \\ Clinical Epidemiology, Oregon Health \& Science University, Portland, OR, USA \\ ${ }^{6}$ Oregon Clinical and Translational Research Institute, Oregon Health \& Science University, \\ Portland, OR, USA \\ ${ }^{7}$ present address: Boundless Bio, La Jolla, CA, USA \\ ${ }^{8}$ Dept of Biomedical Engineering, Oregon Health \& Science University, Portland, OR, USA
}

\begin{abstract}
Invadopodia are actin-based proteolytic membrane protrusions required for invasive behavior and tumor growth. In this study, we used our high-content screening assay to identify kinases whose activity impacts invadopodia formation. Among the top hits selected for further analysis was TAO3, a STE20-like kinase of the GCK subfamily. TAO3 was overexpressed in many human cancers and regulated invadopodia formation in melanoma, breast, and bladder cancers.

Furthermore, TAO3 catalytic activity facilitated melanoma growth in 3-dimensional matrices and in vivo. A novel, potent catalytic inhibitor of TAO3 was developed that inhibited invadopodia formation and function as well as tumor cell extravasation and growth. Treatment with this inhibitor demonstrated that TAO3 activity is required for endosomal trafficking of TKS5a, an obligate invadopodia scaffold protein. A phosphoproteomics screen for TAO3 substrates revealed the dynein subunit protein LIC2 as a relevant substrate. Knockdown of LIC2 or expression of a
\end{abstract}

\footnotetext{
${ }^{9}$ to whom correspondence should be addressed: Oregon Health and Science University, Robertson Collaborative Life Sciences Building (CL6C), 2730 SW Moody Ave, Portland, OR 97201. Telephone: 503-494-4750, courtneidge@ohsu.edu.

these authors contributed equally to the work

Conflict of interest disclosure: The authors declare no potential conflicts of interest.
} 
phosphomimetic form promoted invadopodia formation. Thus, TAO3 is a new therapeutic target with a distinct mechanism of action.

\section{Keywords}

TAO3; TAOK3; invadopodia; TKS5a; endosomes

\section{INTRODUCTION}

Much progress has been made in recent years in the development of novel cancer therapeutics. Among small molecules, kinase inhibitors have met with particular clinical success, with approvals of agents targeting mutated "driver" kinases, as well as non-mutated but essential enzymes [1]. Despite these successes, both intrinsic and acquired resistance limit long-term efficacy. This has led to the development of inhibitors specifically targeting resistance mechanisms, as well as to combination therapies. In the case of mutant B-RAF driven melanoma, combination therapy with the B-RAF inhibitor, dabrafenib, and the MEK inhibitor, trametinib, was recently approved [2-4]. This regimen results in improved survival, although resistance emerges in most patients after approximately 1 year. There are currently no kinase inhibitor strategies for those melanomas with wild-type B-RAF. Exciting progress has also been made in immunotherapy approaches to treat cancer, with noted successes for antibodies targeting PD-1 and CTLA-4 in all subtypes of melanoma [5]. Nevertheless, resistance to these agents also limits long-term efficacy. Thus, it is important to continue to identify new therapeutic approaches for eventual use in combination with existing agents. We reasoned that identifying targets in pathways regulating aspects of the cancer phenotype distinct from cell cycle progression and immune evasion might be such an opportunity.

We decided to focus on the invasive behavior of cancer cells. Invasion is required for cancer cells to move into and out of the bloodstream (intra- and extravasation), and therefore underlies the metastasis that is responsible for most cancer deaths. These steps will have already occurred prior to diagnosis in patients for whom removal of the primary tumor is not curative, and therefore therapeutic intervention for intra- and extravasation may not be beneficial. However, there are many examples where invasive behavior has been linked to tumor growth in both primary and metastatic sites, as well as chemoresistance [6-10]; intervention in these processes would be expected to have therapeutic benefit.

One prominent mechanism by which tumor cells exhibit invasive behavior is by the formation of membrane protrusions known as invadopodia [11-13]. These complex structures coordinate the actin cytoskeleton with pericellular proteolysis, and metallo-, cysteine and serine protease activities have all been described at invadopodia [14]. It has long been known that the presence of invadopodia correlates with invasive behavior in a number of cancer cell types in vitro. More recently, invadopodia were observed in human cancer specimens ex vivo $[15,16]$, and were shown to be required for tumor cell intra- and extravasation in model systems $[17,18]$. Tumor expression analysis has revealed a correlation between high expression of the obligate invadopodia scaffold protein TKS5a and 
worse outcomes in glial-derived tumors, non-small cell lung cancer and breast cancer [6, 19, 20], as well as with increasing stage in prostate cancer and melanoma [7, 21]. Most importantly, the use of 3-dimensional (3D) culture systems and xenograft assays has revealed that invadopodia also promote the growth of tumor cells $[6,7,22]$. While the full mechanistic details of this phenotype remain to be established, it seems likely that the pericellular proteolytic activity associated with invadopodia both remodels the extracellular matrix (ECM) to the benefit of the tumor, as well as processes and activates growth factors and cytokines required to create a growth-promoting microenvironment. For example, the angiogenesis-eliciting vascular endothelial growth factor (VEGF) is processed by metalloproteases into pro-tumorigenic forms [23], and there is some evidence that loss of invadopodia results in reduced tumor-associated VEGF [6].

What might be good therapeutic targets for invadopodia inhibition? The TKS5a scaffold itself, as well as many other key invadopodia proteins, have no catalytic activity, and thus developing drugs against them, while not impossible, would be challenging [24]. Other key players, such as small GTPases, have to-date proven largely intractable to inhibitor strategies. And inhibitors of the matrix metalloproteases have also not met with success to date, perhaps because of redundancy among different protease classes, as well as lack of specificity and potency. Some years ago, we established a high-content screening assay to identify in an unbiased way regulators of invadopodia formation and function [25]. Using a small compound library in a proof-of-principle screen, we identified several small molecules annotated as cyclin-dependent kinase (CDK) inhibitors. We deconvoluted these hits to identify and subsequently validate CDK5 as their target, and provided the mechanism by which CDK5 regulates invadopodia formation [25]. Interestingly, at around the same time it was recognized that CDK5 is over-expressed and indeed frequently amplified in pancreatic cancer, which elaborate invadopodia [26]. Knockdown of CDK5 had no effect on growth of pancreatic cells on tissue culture plastic, but did markedly inhibit the growth of the cells in 3D matrices as well as in vivo, in keeping with a role for CDK5 as an invadopodia regulator [27]. Together these studies support the conclusion that invadopodia inhibition is a viable strategy to reduce tumor growth, and suggest that kinases might be a valuable class of invadopodia targets. Despite the value of kinase inhibitors for cancer therapy generally, and the large target class (there are more than 500 members of the kinome [28]), it is quite remarkable that most research, as well as most target identification and validation [29], and inhibitor development [30], have focused on just a few enzymes. We therefore set out to screen the entire kinome to identify invadopodia regulators.

\section{MATERIALS \& METHODS}

\section{Cell lines}

The C8161.9 human melanoma cell line (RRID:CVCL_0196), the bladder cancer cell lines T24 (RRID:CVCL_0554) and UM-UC-3 (RRID:CVCL_1783) and the breast cancer cell line, Hs578t (RRID:CVCL_0332) were purchased from ATCC. The luciferase-expressing human breast cancer cell line MDA-MB-231-Luc (RRID:CVCL_1D88) was obtained from Xenogen. For these cell lines, we relied on authentication performed by the provider. The WM793 human melanoma cell line was a gift from Dr. Gary G. Chiang (Abbvie, Chicago, 
IL, RRID:CVCL_8787) and authenticated by the OHSU DNA core facility using short tandem repeat analysis. The Src-transformed NIH-3T3 (Src3T3) mouse cell line was generated by the corresponding author (S.A.C.) and has been described before in [31]. Melanoma, breast cancer cell lines and Src3T3 cells were grown in Dulbecco's Modified Eagle's Medium (10-013-CV, Corning) containing 10\% fetal bovine serum (FBS, HyClone and Gibco). T24 was cultured by McCoy's 5a Medium Modified (ATCC) with 10\% FBS. UM-UC-3 was grown in Eagle's Minimum Essential Medium (ATCC) with 10\% FBS. All cell lines were routinely tested for mycoplasma contamination and confirmed negative for mycoplasma species before use. Cell lines were used within 3 months of initiating growth in tissue culture.

\section{Screening and validation}

The high content screening assay for invadopodia formation has been previously described [25]. Briefly, we ran the screen twice, each time in quadruplicate, with a negative control (non-target scrambled siRNA). We used pools of three different siRNAs for each gene. The results from the two runs had a low coefficient of variability, therefore we did not repeat the assay a third time. The effects of siRNA knockdown on invadopodia number were determined by eye, counting at least 100 cells for each field. We considered cells as positive if they had more than two invadopodia. siRNAs that triggered invadopodia inhibition $<50 \%$ (compared to control) in cells that retained viability and otherwise contained a normal actin cytoskeleton were considered hits. In order to validate the screening results, we used the three individual siRNAs (Mission siRNA, Sigma). The validation step was repeated three times and we scored for phenotypic concordance. For each hit, we tested the effect of kinase knockdown on gelatin degradation (using the pool siRNA only). The siRNA oligo IDs are listed in Supplementary Table S1.

\section{Expression analysis}

Cancer Outlier Profile Analysis (COPA) was performed on public gene expression data sets curated by Oncomine [32]. Gene expression values were median-centered, setting each gene's median expression value to zero. The median absolute deviation (MAD) was calculated and scaled to 1 by dividing each gene expression value by its MAD. The 75th, 90th, and 95th percentiles of the transformed expression values were calculated for each gene, and then genes rank-ordered by their percentile scores, providing a prioritized list of outlier profiles. A gene rank over-expression threshold of $10 \%$ was set for this analysis For each gene identified as an outlier in at least one dataset, we tabulated all data sets with outlier calls and the total number of datasets for that cancer type with expression of that gene.

\section{High-resolution invadopodia and time-lapse imaging}

Src3T3 cells were cultured on glass coverslips and prepared for imaging using the same methodology as for the invadopodia assay. Confocal images were collected using a laserscanning confocal microscope LSM880 equipped with AiryScan (Carl Zeiss). Time-lapse imaging was performed by $200 \mathrm{~ms}$ per frame for $1 \mathrm{~min}$, total 300 frames. Images were transferred to Imaris ${ }^{\mathrm{TM}}$ (Bitplane) which is a multidimensional analysis program based on the fluorescence intensity data. For colocalization analysis, surfaces (spots) were created on 
the TKS5, TAO3 and RAB11 separately and colocalized signals were assessed by "ImarisColoc" tool. Detailed methodology is shown in Supplementary Figure S5. For vesicle trafficking, surfaces (spots) were created on the TKS5a-mCherry or RAB11-DsRed to track the vesicles and extract the "displacement length $(\mu \mathrm{m})$ " and "Length $(\mu \mathrm{m})$ " as statistics Excel files. For making movies, the time-lapse films were edited by Apple's iMovie software.

\section{IHC and scoring}

Primary melanomas were stained with TAO3 (ab150388, 1:300, Abcam, RRID:AB_150388) on a Ventana Discovery autostainer. Negative control slides were stained with Rabbit IgG. Subsequently, TAO3 IHC was scored by a board-certified pathologist (G.V.T). Scores were either 0 for no staining in the tumor, $1+$ for weak staining, 2+ for moderate to strong staining. Next, an immunoscore was calculated from the formula: $(0 \mathrm{x} \%$ cells staining negative $)+(1 \times \%$ cells staining weakly positive $)+(2 x \%$ cells staining moderatelystrongly positive), giving a range of $0-200$.

\section{Tumor growth in mice}

All animal experiments were conducted in accordance with the NIH Guide for the Care and Use of Laboratory Animals, and were reviewed by an institutional IACUC. Subcutaneous implantation was performed with minor modifications as described previously [7]. Briefly, cells were resuspended in PBS/Matrigel mixture (1:1 ratio, BD Biosciences) to a final concentration of $4 \times 10^{6}$ cells $/ \mathrm{ml}$. Athymic nude mice (nude Nu/J, The Jackson Laboratory, RRID:IMSR_JAX:002019) were injected in the flank with $100 \mu$ l, and tumors were allowed to form for 22 days. For the tumor growth experiment with inhibitor, SBI-581, tumors were allowed to form for 10 days and SBI-581 (final concentration $10 \mathrm{mg} / \mathrm{kg}$ in DMSO with 1:5 dilution of PBS) were injected every day for 10 days. Tumor growth was measured every 23 days using calipers. The longest (L) and shortest (S) measurements were recorded and tumor volumes were calculated as Volume $=0.5^{*}(\mathrm{~L} \times \mathrm{S} 2)$ and expressed as mean volume $(\mathrm{mm} 2)$.

\section{Extravasation efficiency assay in mice}

Extravasation efficiency assay was performed as described previously [18]. GFP expressing C8161.9 cells (RRID:CVCL_0196) with pLKO.1-scrambled, TAO3 knockdown (KD) or rescued (SR) were injected intravenously in tail veins of 8 week old SCID/Beige female mice (C.B-17/IcrHsd-Prkdc scid Lyst bg-J, ENVIGO). 24 hours after injection of 2.5 x 105 of cells, mice were euthanized and tumor cells in lung blood vessels were removed by perfusion, then lungs were harvested. Frozen serial sections of all lungs (50 $\mu \mathrm{m}$ thickness) were taken every five sections. For the efficiency analysis, slides were counter stained with Hoechst for 15 min before mounting. All sections were scanned by Zeiss AxiaScan system with ZEN Blue software. GFP positive cells were counted from all scanned lung sections then calculated as "Number of GFP+ cancer cells in lung". For inhibitor experiment, DMSO control or SBI-581 $(30 \mathrm{mg} / \mathrm{kg})$ was injected intraperitoneally, then 30 minutes later GFPlabeled C8161.9 cells were injected through tail vein. 


\section{Phosphoproteomic analysis}

C8161.9 cells with endogenous TAO3 replacement by shRNA-TAO3 and wild-type TAO3 (shRNA-resistant, SR) or kinase-dead TAO3 (shRNA-resistant, KR) on five $150 \mathrm{~mm}$ culture dishes grown to between 70-80\% confluence. Cells were washed with $1 \mathrm{X}$ cold-PBS before lysis to remove any media containing protein contaminants. Lyse the cells by $10 \mathrm{ml}$ of Urea Lysis Buffer (at room temperature, $50 \mathrm{mM}$ HEPES pH 8.5, $8 \mathrm{M}$ urea, $1 \mathrm{mM} \mathrm{NaF}, 1 \mathrm{mM}$ sodium orthovanadate, $10 \mathrm{mM}$ sodium $\beta$-pyrophosphate, $1 \mathrm{mM} \beta$-glycerophosphate buffer) and disrupted by sonication on ice. The cell lysates were stored at $-80^{\circ} \mathrm{C}$ prior to digestion and enrichment. Protein concentrations were then determined using the Pierce ${ }^{\mathrm{TM}} \mathrm{BCA}$ Protein Assay Kit (Thermo Scientific). Approximately $12 \mathrm{mg}$ of protein from each lysate was used. Protein was reduced with dithiothreitol, alkylated with iodoacetamide, urea diluted to $2 \mathrm{M}$, then trypsin (TPCK treated, Worthington) was added at a 25:1 protein:trypsin ratio. The samples were incubated overnight at $37^{\circ} \mathrm{C}$ before being quenched with TFA at a final concentration of $1 \%$. The peptides were then solid phase extracted using Waters Sep tC18 cartridges (Waters Corporation). Peptide concentrations were then determined using the Pierce ${ }^{\mathrm{TM}}$ Quantitative Colorimetric Peptide Assay (Thermo Scientific) and dried by vacuum concentration. The phosphopeptides were enriched using previously published methods [33, 34] using Titanosphere $\mathrm{TiO}_{2} 5 \mu \mathrm{m}$ particles (GL Biosciences). The enriched phosphopeptides were purified by solid phase extraction using UltraMicroSpin columns (The Nest Group, Inc.) and dried down in preparation for TMT labeling. The enriched phosphopeptides from the SR and KR cells were then labeled with TMT reagents as recommended by the manufacturer (Thermo Scientific), mixed, and analyzed by twodimensional liquid chromatography/mass spectrometry using an Orbitrap Fusion Tribrid mass spectrometer (Thermo Scientific) as previously described [35]. The full proteomic dataset can be found in the PRIDE database with the following accession number (provided upon publication).

\section{Protein production}

The protein (TAO3 kinase domain (1-319) fused with GST) was expressed in the baculovirus intracellular expression system. Linear DNA was used for the transfection to make virus. Protein was expressed using 8 liters of SF9 cells. After 72 hours of infection, cells were collected by centrifugation, re-dissolved in a buffer containing $50 \mathrm{mM} \mathrm{NaCl}, 20$ mM TRIS pH 8, 1 mM EDTA, $2 \mathrm{mM}$ of $\beta$-mercaptoethanol and including a standard protease inhibitor cocktail. Cells were lysed in this buffer with a glass dounce homogenizer on ice. The supernatant was separated from pellet by centrifugation for $1 \mathrm{~h}$ at $20 \mathrm{~K} \mathrm{rpm}$ (SS34 rotor) and then incubated with glutathione-agarose bead for $2 \mathrm{HRS}$ at $4^{\circ} \mathrm{C}$. Beads were washed with $50 \mathrm{mM} \mathrm{NaCl}, 20 \mathrm{mM}$ TRIS pH 8, $1 \mathrm{mM}$ EDTA, $5 \mathrm{mM} \beta$-ME and then the TAO3 kinase domain was cleaved off the beads by incubation with $3 \mathrm{C}$ protease. Cleaved released kinase domain was separated from contaminating GST by immediately running the sample in this buffer onto a mono-Q on the AKTA FPLC, eluting protein with a gradient of the same buffer containing 50 to $1 \mathrm{M} \mathrm{NaCl}$. The peak containing the TAO3 kinase domain was collected and then run on a Superdex S200 gel filtration column prequilibrated with the final buffer $100 \mathrm{mM} \mathrm{NaCl}, 25 \mathrm{mM}$ Tris-Cl pH 8.0, $2 \mathrm{mM} \beta$-ME, $0.3 \mathrm{mM}$ EDTA. 


\section{High throughput screen for TAO3 Inhibitors}

A time-resolved fluorescence resonance energy transfer (TR-FRET) assay was developed to monitor the inhibition of TAO3 kinase activity. We used the kinase domain of TAO3, and an HTRF-KinEASE (Cisbio) assay along with the $\mathrm{S} 3$ substrate and a $\mathrm{Eu}^{3+}$ Cryptate-conjugated anti-pSer/Thr antibody. This assay was miniaturized to 1536 well format with a final volume of $2 \mu \mathrm{L}+2 \mu \mathrm{L}$ detection reagent. Compounds (including a known kinase inhibitor collection (800 compounds) and an SBP Institute-selected kinase inhibitor scaffold library $(4,800$ compounds)) were tested at $10 \mu \mathrm{M}$. Assay performance was very robust with an average $\mathrm{Z}$ ' of 0.89 , and with no individual plate with a Z' of less than 0.70 . Hit threshold at $30 \%$ inhibition, and since the hit rate was low (not unexpected based on the lack of reported TAO3 inhibitors in the scientific and patent literature) this resulted in 82 hits $(0.12 \%)$. To further prioritize confirmed hits, apparent $\mathrm{IC}_{50}$ values were determined. Twelve selected compounds with potencies $<2 \mu \mathrm{M}$ were validated using Protein Thermal Shift (PTS) technology, which demonstrated direct binding of the compounds to TAO3. These compounds were also tested in dose response against an unrelated kinase (MEKK3) using the same assay format, with a minimum selectivity threshold of 5x. From these efforts a series of oxindoles were identified as the most promising hits. Preliminary chemistry led to SBI-581 $\left(\mathrm{IC}_{50}=42 \mathrm{nM}\right.$ against TAO3, $\mathrm{IC}_{50}=237 \mathrm{nM}$ against MEKK3).

\section{Rodent pharmacokinetics of SBI-581}

SBI-581 was dosed at $1 \mathrm{mg} / \mathrm{kg}$ iv (as a $1 \mathrm{mg} / \mathrm{mL}$ solution in $75 \% \mathrm{PEG} 300 / 25 \%$ water), 10 $\mathrm{mg} / \mathrm{kg}$ po (as a $3 \mathrm{mg} / \mathrm{mL}$ solution in Pharmatek \#6) and $10 \mathrm{mg} / \mathrm{kg}$ ip (as a $3 \mathrm{mg} / \mathrm{mL}$ solution in 5\% DMSO/5\% Tween 80/90\% water) to fasted male C57BL/6 mice (3 mice per cohort, RRID:IMSR_JAX:000664). Plasma samples were taken at 0.083, 0.25, 0.5, 1, 2, 4, 6 and 24 hr post dose (iv) and $0.25,0.5,1,2,4,6$ and $24 \mathrm{hr}$ post dose (po and ip) and measured for the level of SBI-581 via LC/MS/MS analysis.

\section{Quantification and statistical analysis}

The numbers of samples (technical replicate) and times of experiments (biological replicate) are indicated in each figure or figure legend. All data points and error bars represent the means and SEMs. GraphPad Prism software was used to make graph and calculate significance by Student's $t$ test. $P$ value of $<0.05$ was considered to be statistically significant and indicated in figure legends.

Additional information on reagents and assays can be found in Supplementary Materials and Methods.

\section{RESULTS}

\section{Screening for kinases regulating invadopodia formation}

We previously described a high-content image-based screening assay designed to identify regulators of invadopodia formation [25]. Our strategy was to use Src3T3 cells (a mouse model of fibrosarcoma that elaborates abundant invadopodia) for the initial screen. Top hits were subsequently validated in human cancer cell lines. This study led to the identification and validation of CDK5. We used the same assay here. A library of pooled siRNAs (3 for 
each murine kinase [36]), targeting all protein kinases as well as select lipid and metabolic kinases, was kindly provided by Dr. Pam Carroll of Merck. Src3T3 cells, in 1536 well format, were transfected with the pools, incubated for 48 hours, then stained with phalloidin and DAPI to label the F-actin in invadopodia and nuclear DNA respectively. Following automatic well focusing and image capture, each well was analyzed by eye for cell number and viability. For those wells where no cytotoxicity or apoptosis was observed, invadopodia were then evaluated. From this, 12 kinases were prioritized. One of these was the previously validated CDK5, which we did not explore further here. For the others, we next tested each siRNA separately, requiring at least 2 of the 3 to inhibit to proceed. One kinase was deemed off-target by this criterion, leaving 10 in our list (Figure 1A). Representative images for these are shown in Figure 1B and the results summarized in Table 1. Next, we performed a functional assay (gelatin degradation). Knockdown of 3 of these kinases, while inhibiting invadopodia formation, did not affect matrix degradation (Figure 1C, Table 1), and were not pursued further.

Our top hits were thus narrowed to CAMK1, HK2 (a metabolic enzyme), MAP2K1, PAK6, RAF1, TAO3 and TGF $\beta R 2$. Of these, we chose to focus on those kinases we considered understudied, that is with no or few reports to date on involvement in cancer progression and/or invasion. This narrowed our list down to CAMK1, PAK6 and TAO3, all serinethreonine kinases.

\section{Expression analysis of top hits}

The next step was to determine the expression of these 3 kinases in cancer tissue and cell lines. We first performed Cancer Outlier Profile Analysis (COPA) [37] on public gene expression data sets curated by Oncomine [32]. Genes scoring in the top 10\% of COPA scores at any of three percentile cutoffs (75th, 90th, and 95th) were deemed outliers in their respective datasets. At the $10 \%$ gene rank, TAOK3 was an outlier in 28 studies in 17 cancer types, PAK6 was an outlier in 31 studies in 14 cancer types and CAMK1 was an outlier in 44 studies in 16 cancer types. Some of these tumor types (eg melanoma, breast, bladder) have been associated with invadopodia formation in several studies, whereas others (particularly leukemia and lymphoma) have not been well studied. Since it was important that we be able to assess the role of these kinases in human cancer cells in vitro, we chose to focus on melanoma, breast and bladder cancers (Supplementary Table S2, S3, S4).

We first determined the expression of the 3 kinases in representative melanoma, bladder and breast cancer cell lines by qPCR, and for TAO3 and CAMK1 also by immunoblotting (Figure 1D, E). All kinases were expressed in the melanoma cell lines, so we chose to pursue them further in this cancer type. We first used transient RNA interference to reduce expression of each in C8161.9 cells (Supplementary Figure S1A, B), and evaluated both invadopodia formation and function (Figure $1 \mathrm{~F}, \mathrm{G}$ ). The invadopodia formation assay was also performed in another human melanoma cell line, WM793, which confirmed reduction in invadopodia number (Supplementary Figure S1C). In both assays TAO3 knockdown had a more robust inhibitory effect that knockdown of the other kinases. In our screening procedure, we excluded kinases that appeared to reduce cell viability. By using siRNA to knockdown each member of the TAO family in C8161.9 cells, and evaluating the cells 3 
days later, we found that cell number was compromised by loss of TAO1 or TAO2, but loss of TAO3 had no effect (Figure S1D), confirming our screening data.

Finally, we determined the expression of TAO3 at the protein level in clinical specimens of melanoma. An automated immunohistochemical staining protocol was developed then used to stain 20 samples of primary melanoma. The slides were scored (using a $0-2$ scale, where $0=$ negative; 1 =weak; $2=$ mod-strong) and intensity evaluated across each tumor to derive an immunoscore with a range of 0-200. Nineteen of twenty evaluable samples showed staining in some portion of the tumor, with 7 with scores of 101-150 and 8 with scores greater than 151 , suggesting increased expression in melanomas. In some cases, particularly strong staining could be seen at the tumor border, and in disseminating melanoma cells (Supplementary Figure S1E). Together, these analyses led us to nominate TAO3 as our lead kinase.

The gene expression analyses we described earlier suggested that TAO3 was also highly differentially expressed in breast and bladder carcinomas, and was present in cell lines derived from these tumors (Figure 1D, 1E, Supplementary Table S2). To determine the generality of our findings, we therefore tested whether TAO3 was required for invadopodia formation and function in these cells. Both invadopodia formation and gelatin degradation were inhibited (Supplementary Figure S1F, S1G - note that T24 could not be evaluated for gelatin degradation because of matrix tears caused by pulling). In addition, we determined that the TAO3 siRNA did not affect the expression of TAO1 or TAO2 (Supplementary Figure $\mathrm{S} 1 \mathrm{H})$. Together, these data define TAO3 as a regulator of invadopodia formation and invasion, and further reveal its importance in multiple tumor cell types.

\section{Validation of TAO3 in melanoma}

To further validate TAO3 in melanoma, we generated a lentivirus expressing shRNA specific for TAO3, as well as non-targetable expression constructs for wild-type and kinase-dead (via mutation of lysine 53 in the ATP binding site of the catalytic domain) TAO3. Quantitative PCR and immunoblotting demonstrated the specificity of the knockdown and successful reexpression of the constructs (Supplementary Figure S2A, S2B). Furthermore, employing a similar knockdown and re-expression strategy, we found that the catalytic activity of TAO1 was not required to rescue the cytotoxic effects seen upon TAO1 knockdown (Supplementary Figure S2C), which may inform specificity requirements for TAO3 inhibitors (see later). For the TAO3 experiments, analysis of invadopodia formation (Figure 2A) and function (Figure 2B) as well as invasion through matrigel (Figure 2C) in C8161.9 melanoma cells revealed that TAO3 knockdown has a significant inhibitory effect in each case, which could be rescued by re-expression of wild-type but not kinase-inactive TAO3. We have previously shown that reducing the expression of the obligate invadopodia scaffold protein TKS5 has an inhibitory effect on growth in 3D collagen matrices, as well as in vivo $[6,7]$. We next determined the effect of TAO3 knockdown in these same assays. When the cells were cultured on top of a thin layer of type I collagen (collagen-I), no growth differences were seen. However, TAO3 knockdown cells showed a profound growth defect in 3D collagen-I as compared to control cells. Growth was rescued by re-expression of wildtype, but not kinase inactive, TAO3 (Figure 2D). We also observed similar growth 
differences in a tumor spheroid assay (Figure 2E), where spheroid size is directly correlated with cell number [38]. Similar effects on invadopodia formation and 3D growth were seen in a second melanoma cell line, WM793 (Supplementary Figure S2D, S2E).

We next evaluated the effect of TAO3 knockdown in vivo. For this, we used 2 distinct assays. First, it has been reported that extravasation of tumor cells into the lungs requires TKS5-dependent invadopodia formation [18]. To test if this was also the case for TAO3, we introduced GFP into the control, knockdown and rescue cells to mark them, then injected them into the tail veins of immunocompromised mice. One day later, mice were sacrificed, lungs removed and sectioned, then fluorescence microscopy used to enumerate tumor cells. TAO3 knockdown markedly inhibited extravasation, which was restored by re-expression of the wildtype protein (Figure 2F). Next, the control and TAO3 knockdown cells, as well as knockdown cells re-expressing wildtype or kinase-inactive TAO3, were injected subcutaneously into immunocompromised mice and tumor growth evaluated over time. TAO3 knockdown caused a profound inhibition of tumor growth, which was rescued by the wildtype but not kinase-inactive TAO3 (Figure 2G). These data are consistent with the growth inhibitory effects we observed in $3 \mathrm{D}$ cultures.

\section{TAO3 inhibitor identification and testing}

Our rescue studies had shown that the kinase activity of TAO3 was required for its role in invadopodia formation in vitro and tumor progression in vivo. To test the therapeutic potential of TAO3 we therefore initiated a high-throughput screening campaign to identify small molecule inhibitors of the TAO3 kinase domain. Briefly, we screened a number of compound libraries including a set of 800 known kinase inhibitors, as well as 4800 compounds with similar structural features to known kinase inhibitors. We identified a number of hits, of which a series of oxindoles were the most promising, based on potency against the TAO3 kinase domain and moderate selectivity against a broad panel of kinases. A preliminary round of chemistry around the hits identified SBI-581 as a proof of concept compound (Supplementary Figure 3A). It displays good potency against TAO3 $\left(\mathrm{IC}_{50}=42\right.$ $\mathrm{nM}$ ) and moderate selectivity against a broad panel of kinases (Supplementary Table S5). In addition, we measured the pharmacokinetics (PK) of SBI-581 in mice. While oral bioavailability was poor $(\% \mathrm{~F}<5)$, SBI-581 displayed reasonable PK after IP injection $\left(\mathrm{t}_{1 / 2}=1.5 \mathrm{hr} ; \mathrm{AUC}=1202 \mathrm{hr} * \mathrm{ng} / \mathrm{mL} ; \mathrm{Cmax}=\sim 2 \mu \mathrm{M}\right.$ after a $10 \mathrm{mg} / \mathrm{kg}$ dose $)$.

We first used SBI-581 in our short-term assays of invadopodia formation (Figure 3A) and gelatin degradation (Figure 3B). We observed a dose-dependent inhibition in both cases, with an $\mathrm{EC}_{50}$ of $<50 \mathrm{nM}$, a dose that had no effect on. We also evaluated spheroid growth, and observed an inhibitor-dependent decrease after 2 days in 3D culture (Figure 3C). Then we determined the effect of SBI-581 in vivo, using both the extravasation and tumor growth assays described earlier. For the extravasation assay, mice were injected intraperitoneally with $30 \mathrm{mg} / \mathrm{kg}$ SBI-581 (a dose chosen to achieve a projected plasma concentration of $>6$ $\mu \mathrm{M}$, a concentration well in excess of the $\mathrm{IC}_{50}$ for TAO3), then 30 minutes later GFP-labeled melanoma cells were introduced via the tail vein. Extravasated tumor cells in the lung were quantified one day later as described earlier. SBI-581 pre-treatment significantly inhibited extravasation, compared to the DMSO control (Figure 3D). For the tumor growth assay, 
C8161.9 cells were implanted subcutaneously, and 10 days later, when tumors had reached approximately $80 \mathrm{~mm}^{3}$, daily intraperitoneal dosing at $10 \mathrm{mg} / \mathrm{kg}$ was begun. We noted a profound inhibition of tumor growth (Figure 3E) with no significant effect on either body weight (Supplementary Figure S3B) or cell viability, even after several days in culture (Supplementary Figure S3C). Together, these data support the validation of TAO3 as an invadopodia, invasion and growth inhibitor.

\section{TAO3 and endosome trafficking}

It is very important to determine how $\mathrm{TAO} 3$ regulates invadopodia formation, both to support its future clinical development, and to identify biomarkers to track its activity. As a first step, we determined its subcellular localization. In Src3T3 cells (which have rosettes of invadopodia), we noticed that much of the TAO3 signal was not at invadopodia, but rather in a perinuclear location reminiscent of endosomes (Figure 4A). Indeed, GFP-tagged TAO3 expressed in Src3T3 cells co-localized with RAB11, a marker of recycling endosomes (Supplementary Figure S4A) [39, 40]. Endosomes are transported along a microtubule network [41], and co-staining experiments revealed a network of microtubules feeding into the invadopodia (Figure 4A) consistent with other studies [42]; TAO3 was present on these microtubules. We used high-resolution microscopy to confirm that TAO3 and RAB11 colocalized on cytoplasmic microtubules (Figure 4B). There are no reports in the literature on the involvement of RAB11 in invadopodia formation and function. Therefore we next used RNA interference to reduce its expression, which resulted in significant reduction in invadopodia number and gelatin degradation (Figure 4C, Supplementary Figure S4B).

Our next task was to determine how RAB11 and TAO3 might impact invadopodia formation. We thought first of the invadopodia scaffold protein TKS5a [24, 43], since it has an aminoterminal PX domain with specificity for PI3P and PI3,4P 2 [44], and PI3P in particular is highly enriched in endosomes $[45,46]$. Indeed, we have recently found, using highresolution microscopy, that TKS5a is present both at invadopodia and on microtubules ([38], an example shown in Supplementary Figure S4C). Here we show that on microtubules, TKS5a co-localized with both TAO3 and RAB11 (Figure 4D, 4E). Furthermore, inhibition of invadopodia formation by treatment of the cells either with the Src family kinase inhibitor SU11333, or the TAO3 inhibitor SBI-581, promoted TKS5a accumulation at RAB11-positive vesicles (Supplementary Figure S4D). The methodology used is described in Supplementary Figure S5 A-G.

We next determined the effect of the TAO3 inhibitor on endosomal trafficking of TKS5a. First, cells were engineered to express mCherry-labeled TKS5a and YFP-labeled tubulin, and trafficking was confirmed by time-lapse confocal microscopy (Figure 5A, Supplementary Movie 1). Then, either vehicle control (DMSO) or $100 \mathrm{nM} \mathrm{SBI-581} \mathrm{was}$ added to the cells and both time trajectory and vesicle displacement length were evaluated (Figure 5B, C, Supplementary Movie 2). The TAO3 inhibitor had a rapid and profound effect on both properties, reducing the population of motile TKS5a-positive vesicles from $24 \%$ to $6 \%$ of total. To determine if SBI-581 affected all RAB11-positive vesicles, the experiment was repeated using cells expressing DsRed-tagged RAB11. In this case, approximately the same fraction of all vesicles was motile (23\%), and more than half of 
these retained motile characteristics after inhibitor treatment (Figure 5C, Supplementary Movie 3). This effect appeared specific to TAO3 inhibition, since inhibiting Src family kinases with SU11333 was not inhibitory to vesicle motility (Supplementary Figure S6A, Supplementary Movie 4). Finally, SBI-581 shows reasonable selectivity at the very low concentrations we used in this (Supplementary Table S5), but we did note that one such coinhibited kinase was ROCK2, which has noted roles in control of the actin cytoskeleton [47], and is possibly involved in invadopodia activity in response to matrix [48]. To determine if ROCK2 played any role in invadopodia formation and endocytic trafficking of TKS5a, we used the ROCK inhibitor Fasudil. ROCK inhibition affected neither the endocytic trafficking of TKS5a-positive vesicles (Supplementary Figure S6B) nor invadopodia formation (Supplementary Figure S6C).

To determine how TAO3 might impact endocytic trafficking of TKS5a, we next initiated a phosphoproteomics screen, using mass spectrometry to identify and compare phosphopeptides in C8161.9 cells in which the endogenous TAO3 was replaced with either wild-type or kinase-inactive TAO3. Supplementary Table S6 shows the 9 candidates whose phosphorylation was significantly increased in the presence of wild-type TAO3 compared to kinase-dead. Of these, we were particularly interested in cytoplasmic dynein 1 light intermediate chain 2 (LIC2), since dynein complexes act as motors to oppose the actions of kinesin motors to traffic vesicles backwards and forwards along microtubules [49, 50]. In particular, light intermediate chains can recruit the dynein complex to endosomes and lysosomes [51] and also contact adaptors and provide a link to cargo [52]. In keeping with a possible role of LIC2 in TAO3 function, we observed that these two proteins frequently colocalize in the cytoplasm [50.6\% colocalization ( $n=239$ TAO3+ signals in $n=7$ cells)], but not at invadopodia (Figure 6A). We next used knockdown and reconstitution experiments to evaluate the role of LIC2 in invadopodia formation, using both C8161.9 (Figure 6B) and WM793 cells (Supplementary Figure S7A, B). Knockdown of LIC2 resulted in an increase in invadopodia formation, which was rescued by re-introduction of the wild-type protein. In contrast, introduction of a LIC2 molecule mutated at the identified phosphorylation site (S202A) markedly inhibited invadopodia formation, whereas introduction of a "phosphomimetic" form (S202D) promoted invadopodia form to the same extent as LIC2 knockdown. To test whether SBI-581 exerted its effects through preventing TAO3 phosphorylation of LIC2 on S202, we compared the effect of DMSO and SBI-581 on these same cells (Figure 6C and Supplementary Figure S7C). In cells expressing the wild-type protein, SBI-581 inhibited invadopodia formation as expected. However, the inhibitor was without effect in cells lacking LIC2 or expressing the S202A mutant. A small, but significant inhibitory effect was observed in cells expressing S202D LIC2, which may reflect a role for other TAO3 phosphorylation sites on LIC2. Together, these data are consistent with LIC2 acting in an inhibitory fashion in invadopodia formation, and suggest that this inhibition is relieved by phosphorylation of LIC2 on serine 202 by TAO3.

\section{DISCUSSION}

Here we used a high-content screening assay to identify kinases required for invadopodia formation, a process that promotes tumor intravasation and extravasation, as well as tumor growth at both primary and metastatic sites. We identified several kinases already implicated 
in invasive behavior, but we ultimately chose to focus on an "understudied" kinase, TAO3, which we found from public databases to be robustly expressed in breast cancer, bladder cancer and melanoma, each highly invasive tumors known to elaborate invadopodia. We validated a role for TAO3 in invadopodia formation and function in vitro, as well as in extravasation and tumor growth of melanoma in vivo. The phenotypes we observe are in keeping with our invadopodia studies using the obligate scaffold protein Tks5a, which we have previously shown to be involved in invasion and tumor growth $[6,7,22]$, through mechanisms including protease localization and type-I collagen signaling [38]. We note that loss of TAO3 expression was not associated with diminished cell viability in our studies, whereas loss of either TAO1 or TAO2 led to rapid cell death in a kinase-independent fashion. It is important to note that while the $\mathrm{N}$-terminal kinase domains of the three proteins are highly homologous, their C-terminal 600-800 amino acid regions are far more distinct from one another. This raises the possibility that the $\mathrm{C}$-terminal regions but not the kinase activity of TAO1 and TAO2 are essential for cell viability. Lastly, in a previous siRNA study using Hela cells, TAOK3 was identified as a survival gene [53]; it is possible that loss of TAO1 or TAO2 (perhaps by RNAi cross-reaction) underlay the observed phenotype in that case. In Drosophila there is a single TAO gene which is involved in both the JNK pathway and actin-microtubule dynamics [54], whereas in more complex organisms, these functions appear to be mediated by different members of the TAO family.

In the course of our experiments, we found that individual knockdown of the 10 kinases from our primary screen showed that they were necessary for invadopodia formation, but 3 of them (AKT3, PKCO and PYK2) were not required for ECM degradation. To our knowledge, this is the first description of the separation of invasive function from invadopodia formation, and in the future, it will be very interesting to determine the mechanism(s) by which these kinases are involved in invasive behavior.

In order to explore the potential of TAO3 as a drug target, we identified SBI-581 via highthroughput screening of a kinase inhibitor library and preliminary chemistry. SBI-581 shows high potency against $\mathrm{TAO} 3\left(\mathrm{IC}_{50}=42 \mathrm{nM}\right)$, moderate selectivity $(>5-10 \mathrm{x}$ against the majority of a broad panel of kinases), and reasonable pharmacokinetics (PK) in mice using IP injection. It therefore represents a good starting point for future optimization. We note, however, that when tested in cells or in vitro, SBI-581 inhibited TAO3 function without significant cellular toxicity. While this may suggest that some degree of TAO-family specificity has already been achieved with SBI-581 it is more likely that the kinase activities of TAO1 and TAO2 are not required for their survival functions, as suggested by the rescue data in Supplementary Figure S2C.

We made the unexpected discovery that TAO3 localizes to RAB11+ endosomes situated on microtubules, as well as to invadopodia. Indeed, we also found that RAB11 was required for invadopodia formation, identifying a new link between recycling endocytosis and invasive behavior. Importantly, TAO3 inhibition reduced the movement of recycling endosomes, particularly of those vesicles containing TKS5a, thereby inhibiting invadopodia formation. The regulatory process of endocytosis involves the packaging of selected plasma membrane proteins, and subsequent sorting either for eventual destruction in the lysosome, or recycling back to the membrane [55], with GTPases of the Rab family regulating each discrete step 
[56]. Receptors, adhesion proteins and extracellular matrix proteins are all targets for endocytosis, and in turn, signaling from these entities can regulate endocytosis. A link between endocytosis and cancer has long been recognized, whereby, for example, oncogenes can promote recycling of key receptors, as well as decrease surface expression of junctional proteins, to promote cell motility [57-59]. Our data expand these properties to include invasive behavior.

We have begun to identify key TAO3 substrates, and validated the dynein protein LIC2 as a target of TAO3 and a negative regulator of invadopodia formation. This suggests a model in which phosphorylation by TAO3 inhibits retrograde vesicular transport, with the net effect of promoting the anterograde transport of TKS5a-containing vesicles to the plasma membrane where invadopodia formation takes place. The mechanism by which TKS5a is loaded onto and off recycling endosomes is obviously of interest, as is the interaction of TAO3 with endosomes. To this latter point, we note that TAO3, kinesin and dynein complexes all contain coiled-coil domains, which are known mediators of both intra- and intermolecular interactions [60]. In a second, unpublished phosphoproteomic analysis, we identified additional TAO3 phosphorylation sites in LIC2, as well as in components of the kinesin complex. In the future, it will be important to define all of the key TAO3 substrates responsible for the phenotype we observe. Nevertheless, the mechanistic underpinnings of TAO3 loss and the potency of the tumor growth and extravasation inhibition we have observed here, coupled with the lack of detrimental effects of TAO3 loss or inhibition in normal cell types, and the high degree of overexpression of TAO3 in several cancer types, supports the preclinical development and eventual clinical testing of selective TAO3 inhibitors.

\section{Supplementary Material}

Refer to Web version on PubMed Central for supplementary material.

\section{ACKNOWLEDGMENTS}

We thank Devon Kelly for assistance in identification and acquisition of human tumor samples from the OHSU Biolibrary, the OHSU Histopathology and Proteomics Shared Resources for their support, Susanne Heynen-Genel (SBP) for her support in the establishment of the high content screening assay, and Pam Carroll for spearheading the generation of the mouse kinome siRNA library. We acknowledge the expert technical assistance by Stefanie Kaech Petrie and Crystal Chaw in the Advanced Multiscale Microscopy Shared Resource (NIH P30 CA069533), and Larry David, Jennifer Cunliffe, John Klimek, and Phillip Wilmarth in the Proteomics Shared Resource at the OHSU Knight Cancer Institute. We thank the personnel and support from the Cancer Center Structural Biology Core at the Sanford Burnham Prebys Medical Discovery Institute (SBP), and at the Lentivirus Production Cores at SBP and OHSU. This research in the Courtneidge lab was/is supported by R01 CA154002 (S. Iizuka, M. Quintavalle, S. Courtneidge) and R01 CA217625 (S. Iizuka, J. Navarro, K. Gribbin, S. Courtneidge) from the National Cancer Institute, National Institutes of Health, as well as by funds from the Knight Cancer Institute. Research and Shared Resource involvement was supported by National Institutes of Health (NIH) grants R01 CA169172 (G. Thomas), P30 CA069533 (G. Thomas), and P30 CA069533 13S5 (G. Thomas) through the Oregon Health and Science University-Knight Cancer Institute. Proteomic analysis in the OHSU Proteomics Shared Resource was supported by National Institutes of Health (NIH) grants P30 CA069533, P30 EY10572, S10 OD012246, and the OHSU School of Medicine Faculty Innovation Fund. Research at Sanford Burnham Prebys Medical Discovery Institute was supported by R01 CA217625 (M. Abelman, C-T. Ma, E. Sergienko, F-Y. Zeng, I. Pass, C. Hassig, A. Pinkerton). 


\section{REFERENCES}

1. Bhullar KS, Lagaron NO, McGowan EM, Parmar I, Jha A, Hubbard BP, et al., Kinase-targeted cancer therapies: progress, challenges and future directions. Mol Cancer, 2018. 17(1): p. 48. [PubMed: 29455673]

2. Heppt MV, Tietze JK, Graf SA, and Berking C, Combination therapy of melanoma using kinase inhibitors. Curr Opin Oncol, 2015. 27(2): p. 134-40. [PubMed: 25602684]

3. Aladowicz E, Ferro L, Vitali GC, Venditti E, Fornasari L, and Lanfrancone L, Molecular networks in melanoma invasion and metastasis. Future Oncol, 2013. 9(5): p. 713-26. [PubMed: 23647299]

4. Johnson DB and Sosman JA, Therapeutic Advances and Treatment Options in Metastatic Melanoma. JAMA Oncol, 2015. 1(3): p. 380-6. [PubMed: 26181188]

5. Marconcini R, Spagnolo F, Stucci LS, Ribero S, Marra E, Rosa F, et al., Current status and perspectives in immunotherapy for metastatic melanoma. Oncotarget, 2018. 9(15): p. 12452-12470. [PubMed: 29552325]

6. Blouw B, Patel M, Iizuka S, Abdullah C, You WK, Huang X, et al., The invadopodia scaffold protein Tks5 is required for the growth of human breast cancer cells in vitro and in vivo. PLoS One, 2015. 10(3): p. e0121003. [PubMed: 25826475]

7. Iizuka S, Abdullah C, Buschman MD, Diaz B, and Courtneidge SA, The role of Tks adaptor proteins in invadopodia formation, growth and metastasis of melanoma. Oncotarget, 2016. 7(48): p. 78473-78486. [PubMed: 27802184]

8. Li R, Peng C, Zhang X, Wu Y, Pan S, and Xiao Y, Roles of Arf6 in cancer cell invasion, metastasis and proliferation. Life Sci, 2017. 182: p. 80-84. [PubMed: 28625359]

9. Bourguignon LYW, Matrix Hyaluronan-CD44 Interaction Activates MicroRNA and LncRNA Signaling Associated With Chemoresistance, Invasion, and Tumor Progression. Front Oncol, 2019. 9: p. 492. [PubMed: 31293964]

10. Li J, Jin Q, Huang F, Tang Z, and Huang J, Effects of Rab27A and Rab27B on Invasion, Proliferation, Apoptosis, and Chemoresistance in Human Pancreatic Cancer Cells. Pancreas, 2017. 46(9): p. 1173-1179. [PubMed: 28902788]

11. Paterson EK and Courtneidge SA, Invadosomes are coming: new insights into function and disease relevance. Febs j, 2018. 285(1): p. 8-27. [PubMed: 28548369]

12. Hoshino D, Branch KM, and Weaver AM, Signaling inputs to invadopodia and podosomes. J Cell Sci, 2013. 126(Pt 14): p. 2979-89. [PubMed: 23843616]

13. Linder S, Wiesner C, and Himmel M, Degrading devices: invadosomes in proteolytic cell invasion. Annu Rev Cell Dev Biol, 2011. 27: p. 185-211. [PubMed: 21801014]

14. Murphy DA and Courtneidge SA, The 'ins' and 'outs' of podosomes and invadopodia: characteristics, formation and function. Nat Rev Mol Cell Biol, 2011. 12(7): p. 413-26. [PubMed: 21697900]

15. Weaver AM, Cortactin in tumor invasiveness. Cancer Lett, 2008. 265(2): p. 157-66. [PubMed: 18406052]

16. Chen YC, Baik M, Byers JT, Chen KT, French SW, and Diaz B, TKS5-positive invadopodia-like structures in human tumor surgical specimens. Exp Mol Pathol, 2019. 106: p. 17-26. [PubMed: 30439350]

17. Gligorijevic B, Bergman A, and Condeelis J, Multiparametric classification links tumor microenvironments with tumor cell phenotype. PLoS Biol, 2014. 12(11): p. e1001995. [PubMed: 25386698]

18. Leong HS, Robertson AE, Stoletov K, Leith SJ, Chin CA, Chien AE, et al., Invadopodia are required for cancer cell extravasation and are a therapeutic target for metastasis. Cell Rep, 2014. 8(5): p. 1558-70. [PubMed: 25176655]

19. Stylli SS, Luwor RB, Kaye AH, S.T. I, Hovens CM, and Lock P, Expression of the adaptor protein Tks5 in human cancer: prognostic potential. Oncol Rep, 2014. 32(3): p. 989-1002. [PubMed: 24993883]

20. Li CM, Chen G, Dayton TL, Kim-Kiselak C, Hoersch S, Whittaker CA, et al., Differential Tks5 isoform expression contributes to metastatic invasion of lung adenocarcinoma. Genes Dev, 2013. 27(14): p. 1557-67. [PubMed: 23873940] 
21. Burger KL, Learman BS, Boucherle AK, Sirintrapun SJ, Isom S, Diaz B, et al., Src-dependent Tks5 phosphorylation regulates invadopodia-associated invasion in prostate cancer cells. Prostate, 2014. 74(2): p. 134-48. [PubMed: 24174371]

22. Blouw B, Seals DF, Pass I, Diaz B, and Courtneidge SA, A role for the podosome/invadopodia scaffold protein Tks5 in tumor growth in vivo. Eur J Cell Biol, 2008. 87(8-9): p. 555-67. [PubMed: 18417249]

23. Lee S, Jilani SM, Nikolova GV, Carpizo D, and Iruela-Arispe ML, Processing of VEGF-A by matrix metalloproteinases regulates bioavailability and vascular patterning in tumors. J Cell Biol, 2005. 169(4): p. 681-91. [PubMed: 15911882]

24. Saini P and Courtneidge SA, Tks adaptor proteins at a glance. J Cell Sci, 2018. 131(1).

25. Quintavalle M, Elia L, Price JH, Heynen-Genel S, and Courtneidge SA, A cell-based high-content screening assay reveals activators and inhibitors of cancer cell invasion. Sci Signal, 2011. 4(183): p. ra49.

26. Eggers JP, Grandgenett PM, Collisson EC, Lewallen ME, Tremayne J, Singh PK, et al., Cyclindependent kinase 5 is amplified and overexpressed in pancreatic cancer and activated by mutant KRas. Clin Cancer Res, 2011. 17(19): p. 6140-50. [PubMed: 21825040]

27. Feldmann G, Mishra A, Hong SM, Bisht S, Strock CJ, Ball DW, et al., Inhibiting the cyclindependent kinase CDK5 blocks pancreatic cancer formation and progression through the suppression of Ras-Ral signaling. Cancer Res, 2010. 70(11): p. 4460-9. [PubMed: 20484029]

28. Manning G, Whyte DB, Martinez R, Hunter T, and Sudarsanam S, The protein kinase complement of the human genome. Science, 2002. 298(5600): p. 1912-34. [PubMed: 12471243]

29. Fedorov O, Muller S, and Knapp S, The (un)targeted cancer kinome. Nat Chem Biol, 2010. 6(3): p. 166-169. [PubMed: 20154661]

30. $\mathrm{Hu} \mathrm{Y}$, Kunimoto R, and Bajorath J, Mapping of inhibitors and activity data to the human kinome and exploring promiscuity from a ligand and target perspective. Chem Biol Drug Des, 2017. 89(6): p. 834-845. [PubMed: 27933727]

31. Seals DF, Azucena EF Jr., Pass I, Tesfay L, Gordon R, Woodrow M, et al., The adaptor protein Tks5/Fish is required for podosome formation and function, and for the protease-driven invasion of cancer cells. Cancer Cell, 2005. 7(2): p. 155-65. [PubMed: 15710328]

32. Rhodes DR, Yu J, Shanker K, Deshpande N, Varambally R, Ghosh D, et al., ONCOMINE: a cancer microarray database and integrated data-mining platform. Neoplasia, 2004. 6(1): p. 1-6. [PubMed: 15068665]

33. Kettenbach AN and Gerber SA, Rapid and reproducible single-stage phosphopeptide enrichment of complex peptide mixtures: application to general and phosphotyrosine-specific phosphoproteomics experiments. Anal Chem, 2011. 83(20): p. 7635-44. [PubMed: 21899308]

34. Paulo JA, McAllister FE, Everley RA, Beausoleil SA, Banks AS, and Gygi SP, Effects of MEK inhibitors GSK1120212 and PD0325901 in vivo using 10-plex quantitative proteomics and phosphoproteomics. Proteomics, 2015. 15(2-3): p. 462-73. [PubMed: 25195567]

35. Wakeham CM, Wilmarth PA, Cunliffe JM, Klimek JE, Ren G, David LL, et al., Identification of PKCalpha-dependent phosphoproteins in mouse retina. J Proteomics, 2019. 206: p. 103423. [PubMed: 31255707]

36. Toyoshima M, Howie HL, Imakura M, Walsh RM, Annis JE, Chang AN, et al., Functional genomics identifies therapeutic targets for MYC-driven cancer. Proc Natl Acad Sci U S A, 2012. 109(24): p. 9545-50. [PubMed: 22623531]

37. MacDonald JW and Ghosh D, COPA--cancer outlier profile analysis. Bioinformatics, 2006. 22(23): p. 2950-1. [PubMed: 16895932]

38. Iizuka S, Leon RP, Gribbin KP, Zhang Y, Navarro J, Smith R, et al., Crosstalk between invadopodia and the extracellular matrix. European Journal of Cell Biology, 2020. 99(7): p. 151122. [PubMed: 33070041]

39. Welz T, Wellbourne-Wood J, and Kerkhoff E, Orchestration of cell surface proteins by Rab11. Trends Cell Biol, 2014. 24(7): p. 407-15. [PubMed: 24675420]

40. Schonteich E, Wilson GM, Burden J, Hopkins CR, Anderson K, Goldenring JR, et al., The Rip11/ Rab11-FIP5 and kinesin II complex regulates endocytic protein recycling. J Cell Sci, 2008. 121(Pt 22): p. 3824-33. [PubMed: 18957512] 
41. Bonifacino JS and Neefjes J, Moving and positioning the endolysosomal system. Curr Opin Cell Biol, 2017. 47: p. 1-8. [PubMed: 28231489]

42. Schoumacher M, Goldman RD, Louvard D, and Vignjevic DM, Actin, microtubules, and vimentin intermediate filaments cooperate for elongation of invadopodia. J Cell Biol, 2010. 189(3): p. 54156. [PubMed: 20421424]

43. Lock P, Abram CL, Gibson T, and Courtneidge SA, A new method for isolating tyrosine kinase substrates used to identify fish, an SH3 and PX domain-containing protein, and Src substrate. EMBO J, 1998. 17(15): p. 4346-57. [PubMed: 9687503]

44. Abram CL, Seals DF, Pass I, Salinsky D, Maurer L, Roth TM, et al., The adaptor protein fish associates with members of the ADAMs family and localizes to podosomes of Src-transformed cells. J Biol Chem, 2003. 278(19): p. 16844-51. [PubMed: 12615925]

45. Schink KO, Raiborg C, and Stenmark H, Phosphatidylinositol 3-phosphate, a lipid that regulates membrane dynamics, protein sorting and cell signalling. Bioessays, 2013. 35(10): p. 900-12. [PubMed: 23881848]

46. Gillooly DJ, Morrow IC, Lindsay M, Gould R, Bryant NJ, Gaullier JM, et al., Localization of phosphatidylinositol 3-phosphate in yeast and mammalian cells. Embo J, 2000. 19(17): p. 457788. [PubMed: 10970851]

47. Julian L and Olson MF, Rho-associated coiled-coil containing kinases (ROCK): structure, regulation, and functions. Small GTPases, 2014. 5: p. e29846. [PubMed: 25010901]

48. Jerrell RJ and Parekh A, Matrix rigidity differentially regulates invadopodia activity through ROCK1 and ROCK2. Biomaterials, 2016. 84: p. 119-129. [PubMed: 26826790]

49. Sweeney HL and Holzbaur ELF, Motor Proteins. Cold Spring Harb Perspect Biol, 2018. 10(5).

50. Holzbaur EL and Vallee RB, DYNEINS: molecular structure and cellular function. Annu Rev Cell Biol, 1994. 10: p. 339-72. [PubMed: 7888180]

51. Tan SC, Scherer J, and Vallee RB, Recruitment of dynein to late endosomes and lysosomes through light intermediate chains. Mol Biol Cell, 2011. 22(4): p. 467-77. [PubMed: 21169557]

52. Reck-Peterson SL, Redwine WB, Vale RD, and Carter AP, The cytoplasmic dynein transport machinery and its many cargoes. Nat Rev Mol Cell Biol, 2018. 19(6): p. 382-398. [PubMed: 29662141]

53. MacKeigan JP, Murphy LO, and Blenis J, Sensitized RNAi screen of human kinases and phosphatases identifies new regulators of apoptosis and chemoresistance. Nat Cell Biol, 2005. 7(6): p. 591-600. [PubMed: 15864305]

54. King I and Heberlein U, Tao kinases as coordinators of actin and microtubule dynamics in developing neurons. Commun Integr Biol, 2011. 4(5): p. 554-6. [PubMed: 22046460]

55. Conner SD and Schmid SL, Regulated portals of entry into the cell. Nature, 2003. 422(6927): p. 37-44. [PubMed: 12621426]

56. Wandinger-Ness A and Zerial M, Rab proteins and the compartmentalization of the endosomal system. Cold Spring Harb Perspect Biol, 2014. 6(11): p. a022616. [PubMed: 25341920]

57. Mellman I and Yarden Y, Endocytosis and cancer. Cold Spring Harb Perspect Biol, 2013. 5(12): p. a016949. [PubMed: 24296170]

58. Schmid SL, Reciprocal regulation of signaling and endocytosis: Implications for the evolving cancer cell. J Cell Biol, 2017. 216(9): p. 2623-2632. [PubMed: 28674108]

59. Rainero E, Extracellular matrix endocytosis in controlling matrix turnover and beyond: emerging roles in cancer. Biochem Soc Trans, 2016. 44(5): p. 1347-1354. [PubMed: 27911717]

60. Mason JM and Arndt KM, Coiled coil domains: stability, specificity, and biological implications. Chembiochem, 2004. 5(2): p. 170-6. [PubMed: 14760737] 


\section{SIGNIFICANCE}

An unbiased screening approach identifies TAO3 as a regulator of invadopodia formation and function, supporting clinical development of this class of target. 

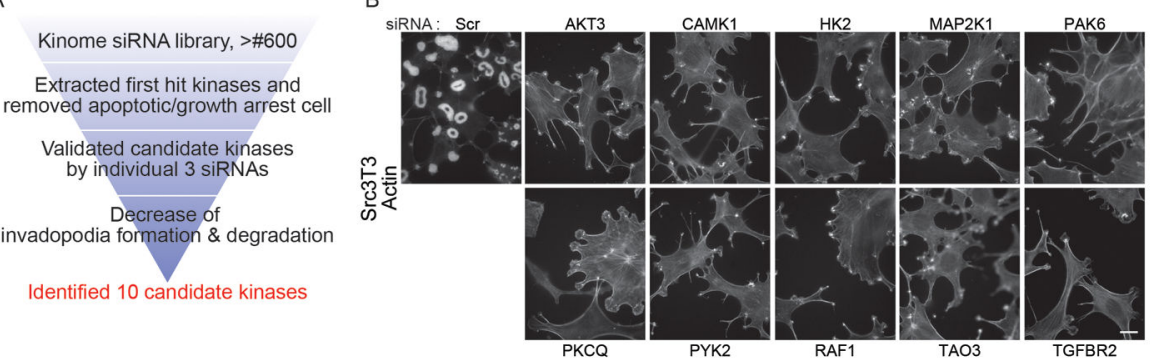

C

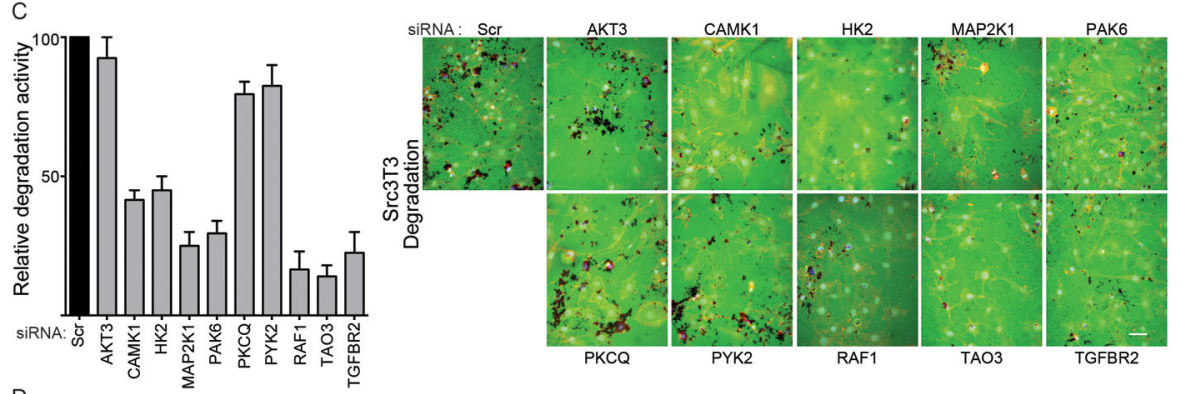

D
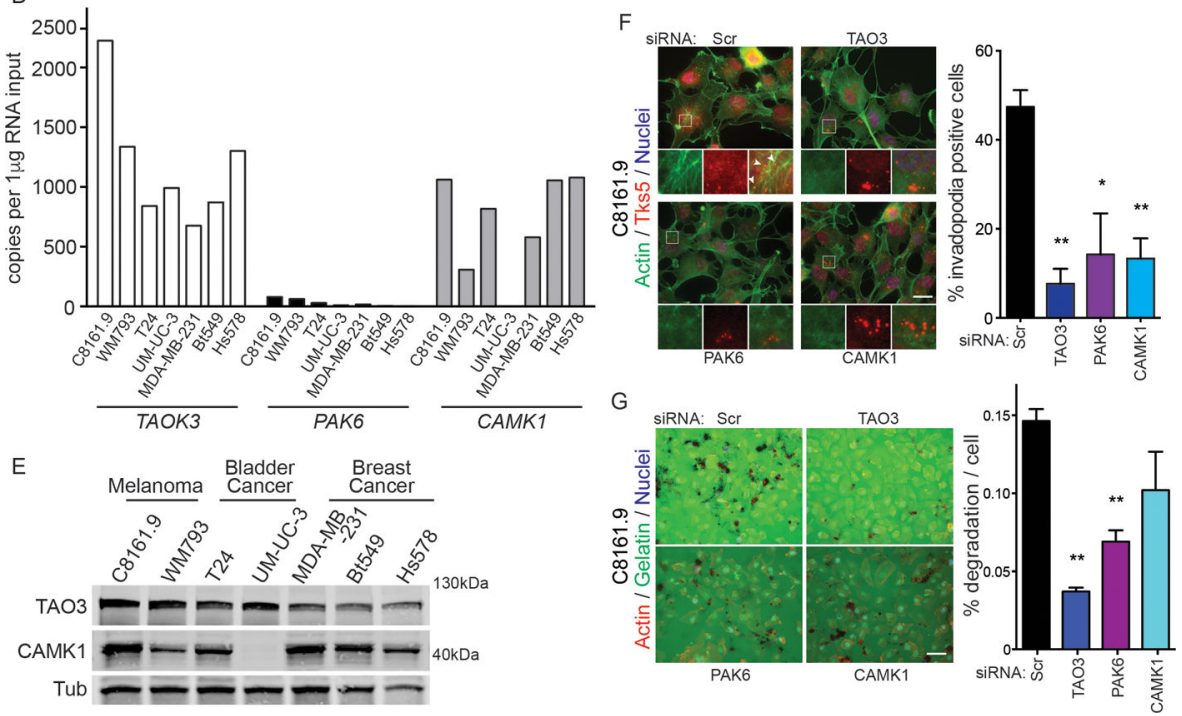

Figure 1. High-content screening image analysis using a kinome siRNA library reveals invadopodia regulators and validation of candidate kinases.

A Schematic view of screening and validation steps. $\mathbf{B}$ and $\mathbf{C}$ Validation analysis of candidate kinases in Src3T3 cells. Invadopodia formation (B) and gelatin degradation (C) with siRNA-scrambled and other 10 candidate kinases. Data shown are representative images and relative degradation activity $(\mathbf{C}$, graph). Immunofluorescence staining of invadopodia for actin (phalloidin in B and C) and gelatin (green in C). D and E Expression of top hit kinases. qPCR (D) and immunoblotting (E) analysis of TAO3, PAK6 and CAMK1 in melanoma, bladder cancer and breast cancer cell lines. Tubulin is shown as a loading control. Invadopodia formation $(\mathbf{F})$ and gelatin degradation $(\mathbf{G})$ analysis in C8161.9 cells with siRNA-scrambled, -TAO3, -PAK6 or-CAMK1. Representative images (left) and percentage of invadopodia positive cells or percentage of degradation per cells (right). Immunofluorescence staining of invadopodia for actin (phalloidin, green in $\mathrm{F}$ and red in $\mathrm{G}$ ) and TKS5 (red in F), gelatin (green in G) and Hoechst (blue) to visualize nuclei. Scale bars, 


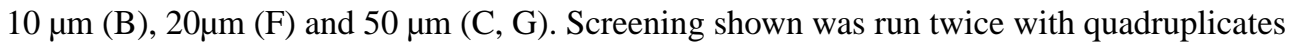
each time. qPCR data shown are technical duplicates and were validated in 2 separated experiments. Immunoblotting data shown was validated in 2 separate experiments.

Invadopodia and degradation assay shown in $\mathrm{F}$ and $\mathrm{G}$ is $\mathrm{n}=5$ to 7 in each group and 2 or more biological replicates. $P>0.05$ unless other specified; *, $P<0.05$; **, $P<0.01$. 


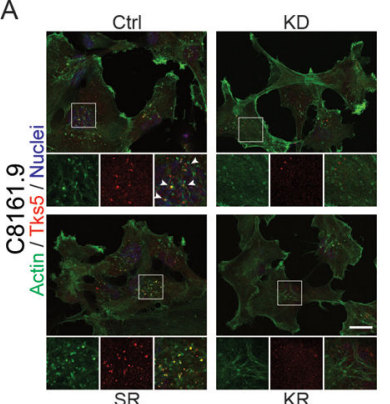

C
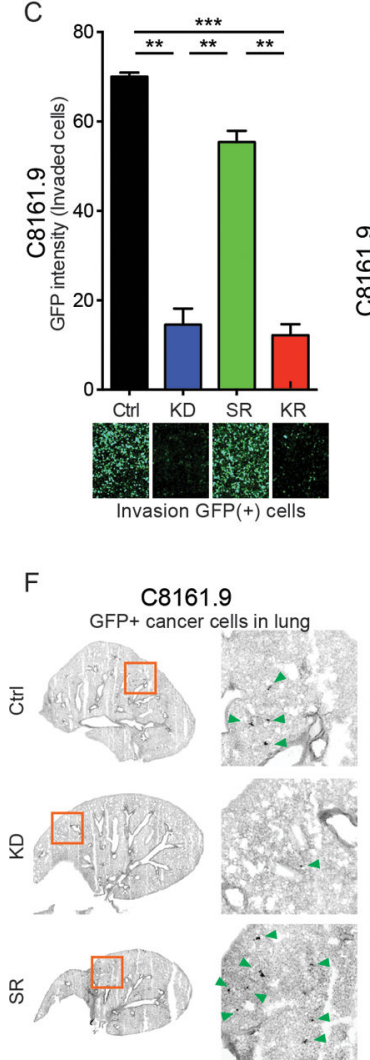

$\mathrm{D}$

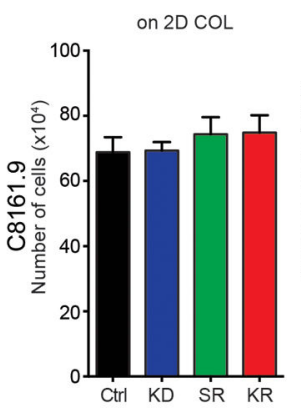

B

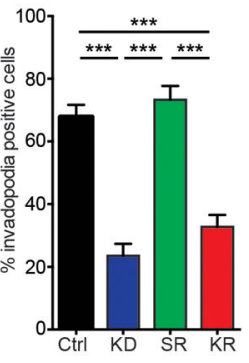

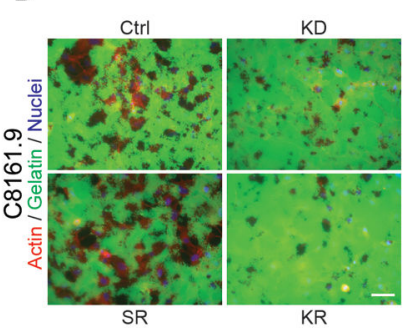

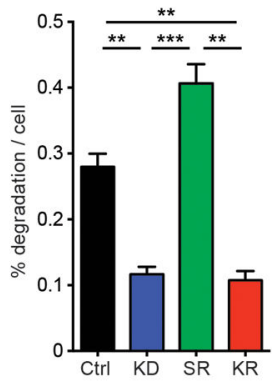

$E$
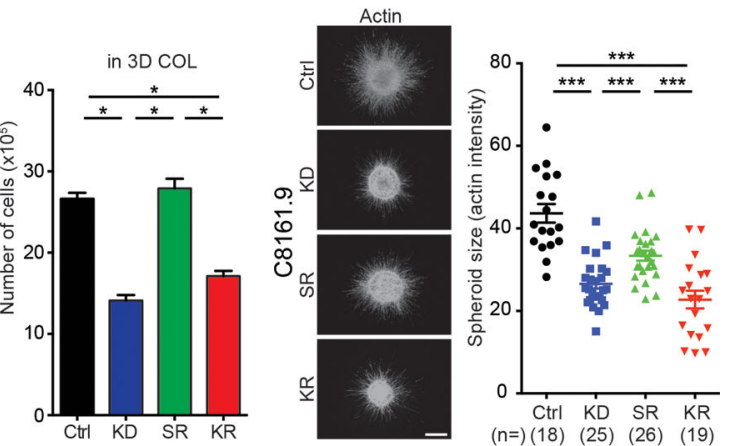
$(n=)(18)(25)(26) \quad(19)$
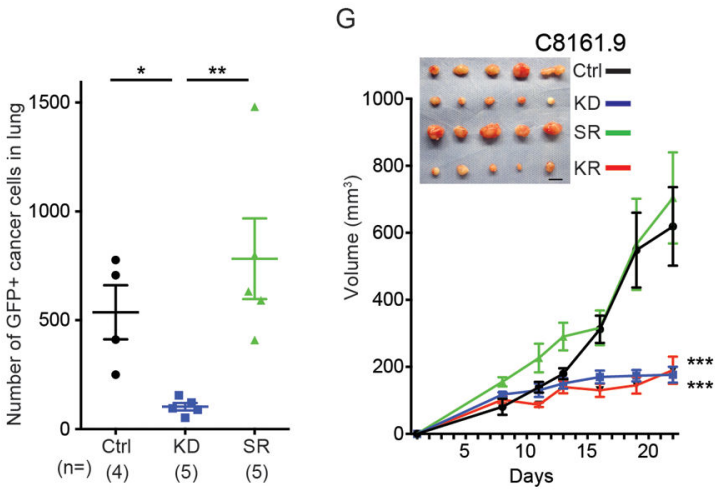

Figure 2. TAO3 is a regulator of invadopodia formation, invasion and growth in 3D conditions. $\mathbf{A}$ and $\mathbf{B}$, Invadopodia formation $(\mathbf{A})$ and gelatin degradation $(\mathbf{B})$ analysis in C8161.9 cells with shRNA-scrambled (Scr), shRNA-TAO3 (KD), shRNA-TAO3+rescued expression of shRNA-resistant TAO3 (SR) or shRNA-TAO3+rescued expression of shRNA-resistant kinase-dead TAO3 (KR). Representative images (left) and percentage of invadopodia positive cells or percentage of degradation per cells (right). Immunofluorescence staining of invadopodia for actin (phalloidin, green) and TKS5 (red), and Hoechst (blue) to visualize nuclei. C Transwell chamber invasion assay with matrigel in the cells as indicated. Graph of GFP signal intensity from invaded cells (top) and representative GFP positive cells (bottom) on bottom of the membrane. D Growth of cells as indicated in the figure on 2D type I collagen (on 2D COL, day 8) and in 3D type I collagen (in 3D COL, day 12). E 3D growth/ invasion in a hanging droplet spheroid assay. Representative images of spheroids in type I collagen stained by phalloidin (left) and spheroid size measured by actin intensity (right). F 
Extravasation efficiency assay in mice. Representative images of GFP+ cancer cells in lung (left) and number of GFP+ cancer cells in lung (right). Note: Blood vessels in lung tissue have high GFP background signal. G Tumor growth in mice injected subcutaneously.

Macroscopic view of all tumors is shown (top). Scale bars, $20 \mu \mathrm{m}$ (A), $50 \mu \mathrm{m}$ (B) and 500 $\mu \mathrm{m}$ (E). Data shown are $\mathrm{n}=3$ to 9 in each experimental group (unless other specified in figure) and were validated in 2 or more separate experiments. $P>0.05$ unless other specified; $*, P<0.05 ; * *, P<0.01 ; * * *, P<0.001$. 
A $\quad$ C8161.9

DMSO

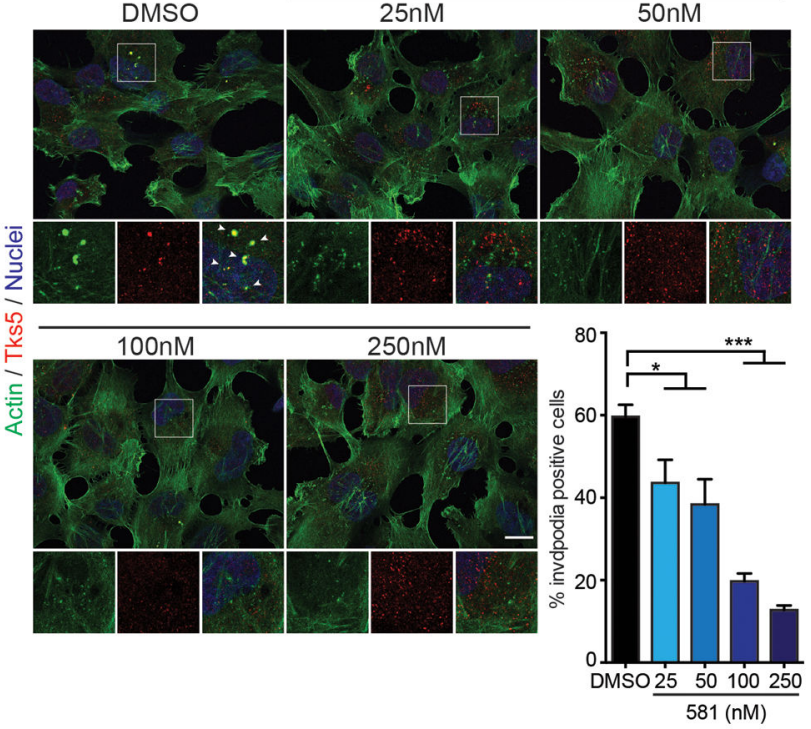

C8161.9

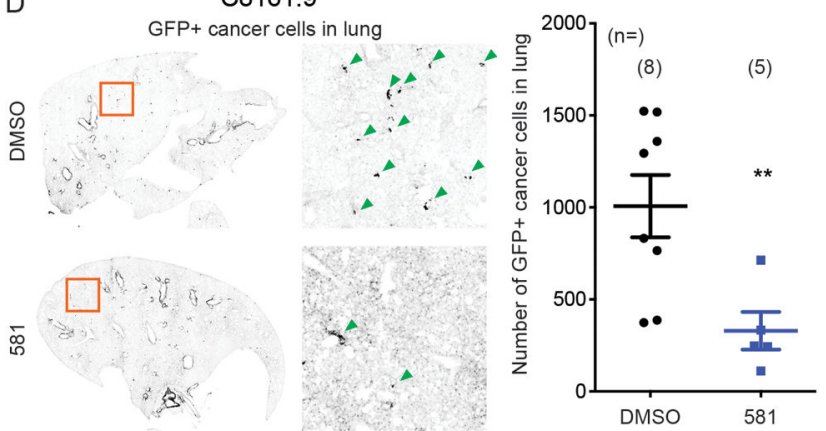

B C8161.9
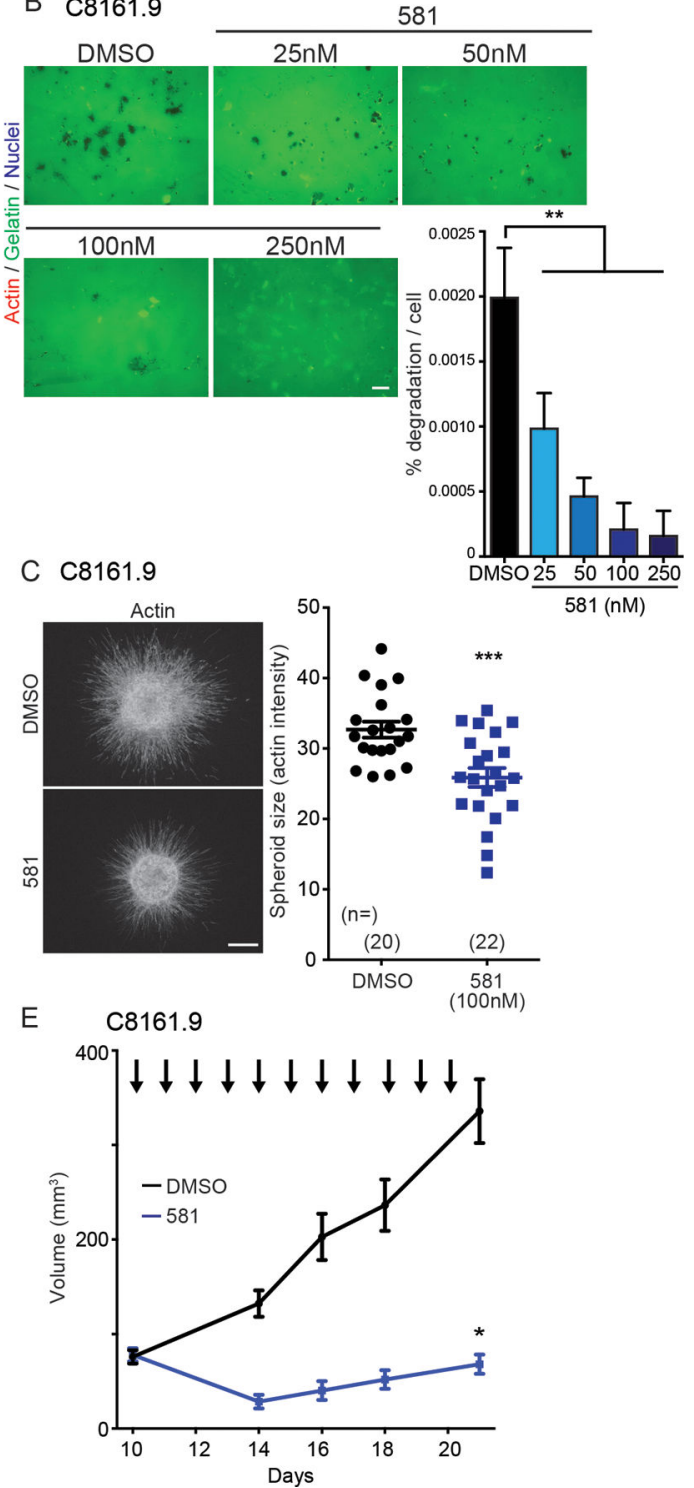

Figure 3. TAO3 inhibitor treatment reduces invadopodia function, 3D growth and extravasation in vitro and in vivo.

$\mathbf{A}$ and $\mathbf{B}$, Invadopodia formation $(\mathbf{A})$ and gelatin degradation $(\mathbf{B})$ analysis in C8161.9 cells with control DMSO and TAO3 inhibitor, SBI-581 (581). Representative images (top left) and percentage of invadopodia positive cells or percentage of degradation per cells (bottom right). Immunofluorescence staining of invadopodia for actin (phalloidin, green) and TKS5 (red), and Hoechst (blue) to visualize nuclei. C 3D growth/invasion by hanging droplet spheroid assay. Representative images of spheroid in type I collagen stained by phalloidin (left) and spheroid size measured by actin intensity (right). D Extravasation efficiency assay in mice. Representative images of GFP+ cancer cells in lung (left) and number of GFP+ cancer cells in lung (right). Note: Blood vessels in lung tissue have high GFP background signal. Scale bars, $20 \mu \mathrm{m}$ (A), $50 \mu \mathrm{m}$ (B) and $500 \mu \mathrm{m}$ (C). Data shown are $\mathrm{n}=6$ to 16 in each experimental group (unless otherwise specified in figure) and were validated in 2 or more separate experiments. E. Tumor growth in mice. Treatment began 10 days after tumor cell 
implantation subcutaneously, with DMSO vehicle or SBI-581 treatment occurring at the time indicated by the arrows. Calipers were used to measure tumor size. $P>0.05$ unless other specified; *, $P<0.05$; **, $P<0.01$; ***, $P<0.001$. 
A

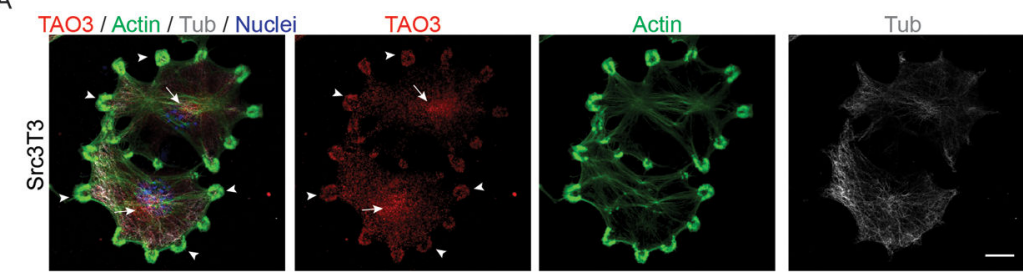

B
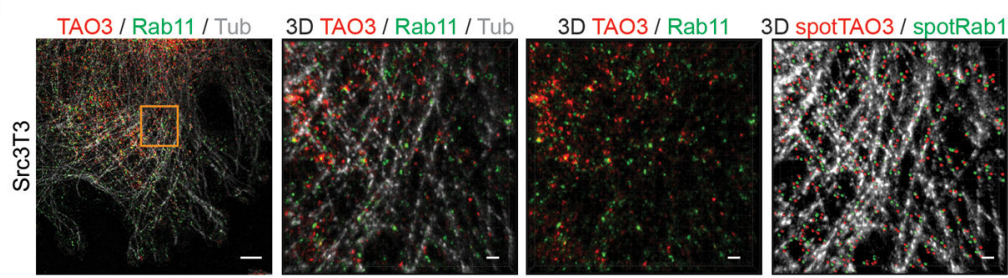

C
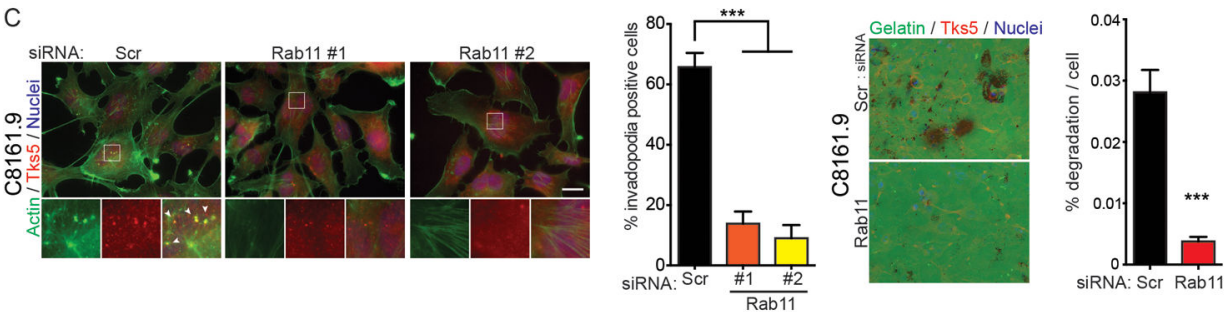

D
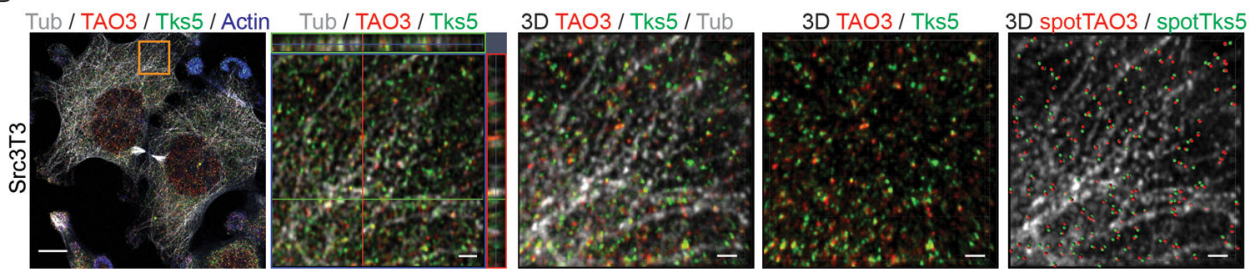

E Rab11 / TAO3 / Tks5 / Tub
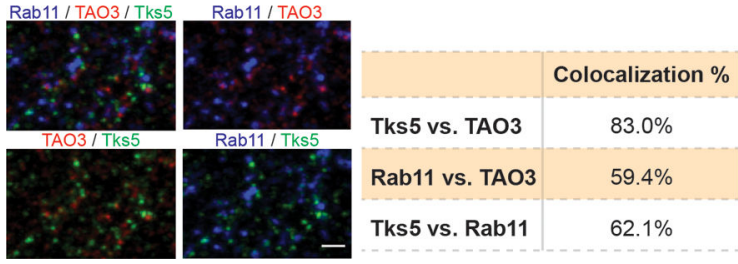

Figure 4. TAO3 localizes at RAB11+ endosomal vesicles with TKS5.

A Distribution of TAO3. Staining of TAO3 (red), actin (green), tubulin (gray) and nuclei

(blue in merged image only) in Src3T3 cells. Images were processed by maximum intensity projection. Arrowheads indicate invadopodial positioning of TAO3. Arrows indicate endosomal positioning of TAO3. B Colocalization of TAO3 and RAB11. Staining of TAO3 (red), RAB11 (green) and tubulin (gray) in Src3T3 cells. Images were processed by maximum intensity projection (left) and 3D reconstruction using Imaris software (3D, magnified area from orange square in left image). Colocalized TAO3 and RAB11 were spotted and shown with tubulin (3D spot, right). C Invadopodia formation (left) and gelatin degradation (right) analysis in C8161.9 cells with siRNA-scrambled and -RAB11. Two individual siRNA-RAB11 (\#1 and \#2) were used for invadopodia assay. Pool-siRNARAB11 (four siRNAs) was used for degradation assay. Representative images (left) and percentage of invadopodia positive cells or percentage of degradation per cells (right). 
Immunofluorescence staining of invadopodia for actin (phalloidin, green) and TKS5 (red), and Hoechst (blue) to visualize nuclei. D Colocalization of TAO3 and TKS5. Staining of tubulin (gray), TAO3 (red), TKS5 (green) and actin (blue) in Src3T3 cells. Images were processed by maximum intensity projection (left). Magnified area (orange square in left) was shown as orthogonal view (second left) and 3D reconstruction by Imaris software (3D). Colocalized TAO3 and TKS5 were spotted and shown with tubulin (3D spot, right). E Colocalization of RAB11, TAO3 and TKS5. Staining of tubulin (gray), RAB11 (blue), TAO3 (red) and TKS5 (green) in Src3T3 cells. Images were processed by maximum intensity projection (left). Magnified area (orange square in left) shown (second left, single plane z-stack image) and higher magnification images are shown in small four panels with different combination of channels. Colocalization between each RAB11, TAO3 and TKS5 was analyzed by Imaris software and shown in table (right). Scale bars, $10 \mu \mathrm{m}$ (A, B left, D left and $\mathrm{E}$ left), $1 \mu \mathrm{m}$ (B and D right three), $2 \mu \mathrm{m}$ (E second left) and $20 \mu \mathrm{m}$ (C). Data shown in panel $\mathrm{C}$ are $\mathrm{n}=6$ to 7 in each experimental group and were validated in two separate experiments. $P>0.05$ unless other specified; $* * *, P<0.001$. 
Color-coded

time projection
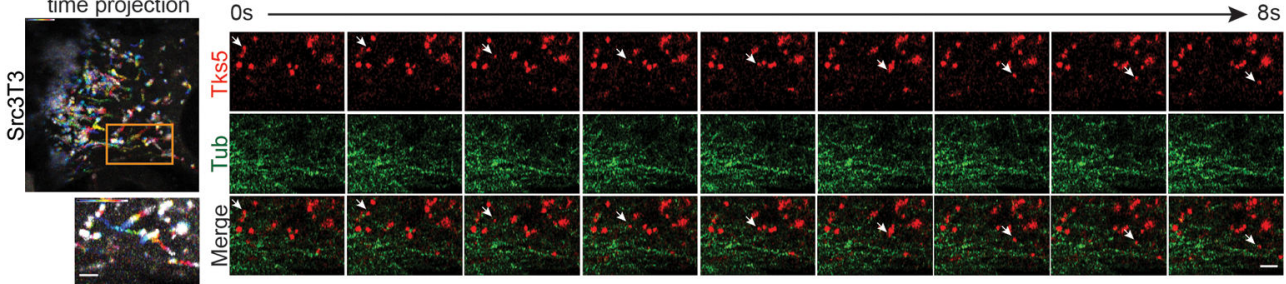

B Color-coded
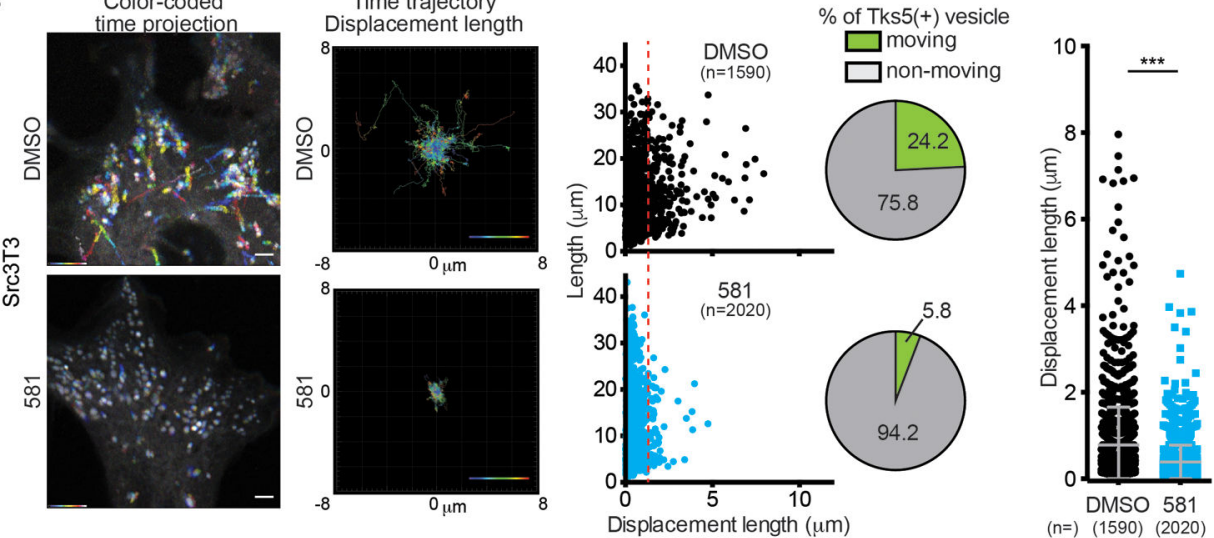

C
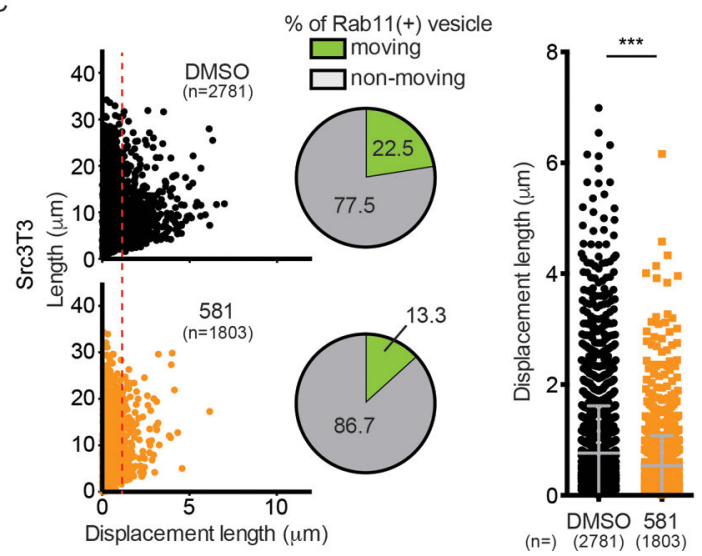

Figure 5. TAO3 regulates trafficking of vesicles containing TKS5.

A Trafficking of TKS5-positive vesicles was captured by time-lapse imaging (200ms/image for $1 \mathrm{~min}$, total 300 images/film) in Src3T3 cells expressing TKS5-mCherry and YFP-

Tubulin. Movie was processed for color-coded time projection (top left) and magnified area (orange square) was shown as color-coded time projection (bottom left) and separated channels in time frame (selected time point during 0-8 sec). B and C SBI-581 inhibits TKS5-positive (B) and Rab11-positive (C) vesicle trafficking. The movies were taken from cells with treatment of DMSO or SBI-581 (100nM) for $1 \mathrm{~min}$ (200ms interval). Trafficking of all TKS5-positive and Rab11-positive vesicles was shown by color-coded time projection (left), time trajectory displacement length (second left), plotting graph of length/ displacement length (middle) with percentage of TKS5+ vesicle moving (pie chart) and displacement length (right). Scale bars $2 \mu \mathrm{m}$ (A, B and C). Data shown in panel B is mean 
SEM of biological replicates from 2 or more separated experiments. $P>0.05$ unless other specified; ***, $P<0.001$. 
A $\mathrm{TAO} 3 / \mathrm{LIC} 2 /$ Actin

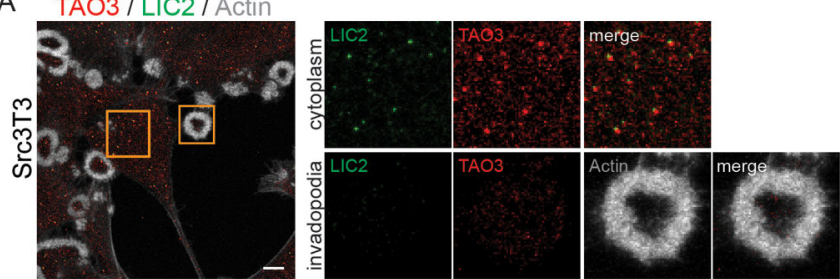

B C8161.9

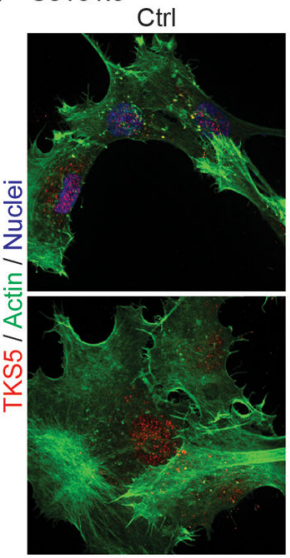

LIC2KD+T202A

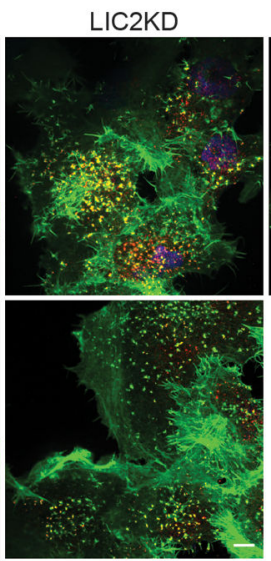

$\mathrm{LIC} 2 \mathrm{KD}+\mathrm{T} 202 \mathrm{D}$
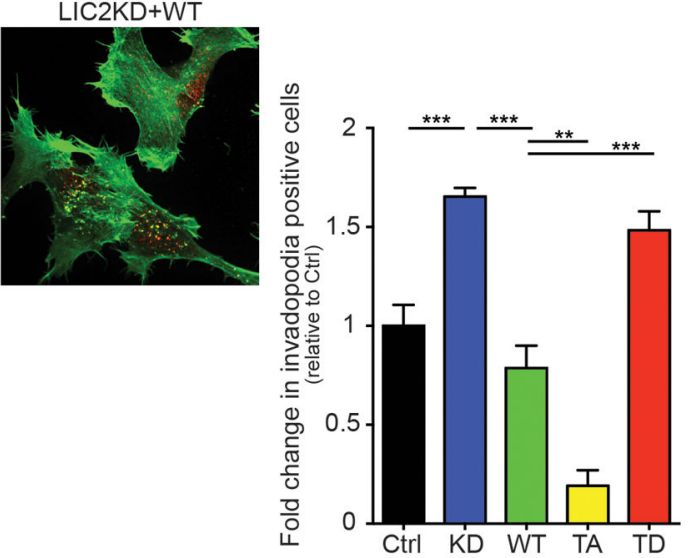

C $\mathrm{C} 8161.9$

TKS5 / Actin / Nuclei

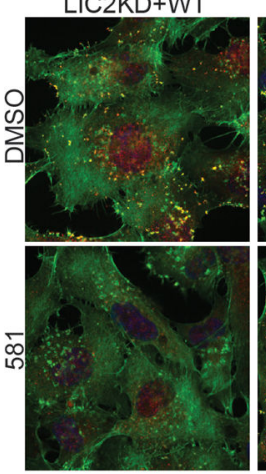

LIC2KD

LIC2KD+T202A
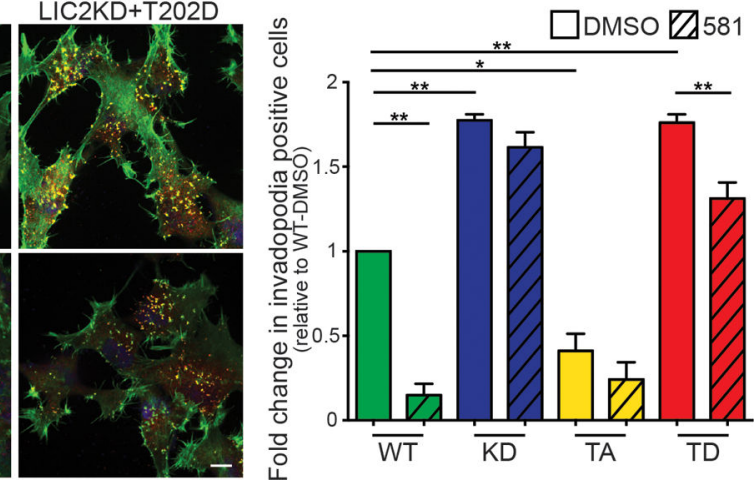

Figure 6. TAO3 phosphorylation of LIC2 promotes invadopodia formation.

A Colocalization of TAO3 and LIC2. Staining of TAO3 (red), LIC2 (green) and actin (gray) in Src-3T3 cells. Images were processed by maximum intensity projection. Magnified area from orange squares (cytoplasm or invadopodia) are shown at right. B Invadopodia formation analysis in C8161.9 cells with shRNA-scrambled (Ctrl), shRNA-LIC2 (LIC2KD), shRNA-LIC2+rescued expression with shRNA-resistant LIC2 wild-type (LIC2KD+WT), LIC2-T202A (LIC2KD+T202A) or LIC2-T202D (LIC2KD+T202KD). Representative images (left) and fold change in invadopodia positive cells (right). Immunofluorescence staining of invadopodia for actin (phalloidin, green) and TKS5 (red), and Hoechst (blue) to visualize nuclei. C. Cells expressing the constructs described in B evaluated for invadopodia formation in the presence of DMSO or SBI-581 $(581,100 \mathrm{nM})$. 
Table 1.

Summary of screening for kinases regulating invadopodia formation and function.

Results for the top 10 hits are shown. $+=$ inhibition $\mathrm{x}=$ no inhibition

\begin{tabular}{|c|c|c|c|c|c|c|}
\hline & \multicolumn{5}{|c|}{ Invadopodia formation assay } & Degradation assay \\
\hline Gene & Protein & pooled siRNA & siRNA \#1 & siRNA\#2 & siRNA\#3 & pooled siRNA \\
\hline CAMK1 & $\begin{array}{l}\text { Calcium/calmodulin-dependent protein kinase } \\
\text { type I }\end{array}$ & + & + & + & + & + \\
\hline MAP2K1 & Mitogen-activated protein kinase kinase 1 & + & $\mathrm{x}$ & + & + & + \\
\hline PAK6 & PAK6 & + & + & + & + & + \\
\hline RAF1 & $\mathrm{cRAF}$ & + & + & + & + & + \\
\hline TAOK3 & TAO3 & + & + & + & + & + \\
\hline TGFBR2 & TGF $\beta$ receptor type 2 & + & + & + & $\mathrm{x}$ & + \\
\hline AKT3 & AKT3 & + & $\mathrm{x}$ & + & + & $\mathrm{x}$ \\
\hline PRKCQ & Protein kinase $\mathrm{C} \Theta$ & + & + & + & + & $\mathrm{x}$ \\
\hline PTK2 & PYK2 & + & + & + & + & $\mathrm{x}$ \\
\hline HK2 & Hexokinase-2 & + & + & + & + & + \\
\hline
\end{tabular}

